\title{
Ultrasound Assessment Of Diaphragmatic
}

\section{Function During Acute Exacerbation Of Chronic Obstructive Pulmonary Disease: A Pilot Study}

This article was published in the following Dove Press journal:

International Journal of Chronic Obstructive Pulmonary Disease

\author{
Sung Yoon Lim' \\ Gajin Lim $^{2}$ \\ Yeon Joo Lee' \\ Young Jae Cho (D) \\ Jong Sun Park' \\ Ho II Yoon ${ }^{1,2}$ \\ Jae Ho Lee ${ }^{1,2}$ \\ Choon-Taek Lee ${ }^{1,3}$ \\ 'Division of Pulmonology and Critical \\ Care Medicine, Department of Internal \\ Medicine, Seoul National University \\ Bundang Hospital, Seongnam, Korea; \\ ${ }^{2}$ Division of Pulmonology Medicine, \\ Department of Internal Medicine, \\ Cheongju Hana Hospital, Cheongju-si, \\ Korea; ${ }^{3}$ Division of Pulmonology and \\ Critical Care Medicine, Department of \\ Internal Medicine, Seoul National \\ University College of Medicine, Seoul, \\ Korea
}

Purpose: Impairment of diaphragmatic function is one of the main pathophysiological mechanisms of chronic obstructive pulmonary disease (COPD) and is known to be related to acute exacerbation. Ultrasonography (US) allows for a simple, non-invasive assessment of diaphragm kinetics. The purpose of this study was to investigate the changes in diaphragmatic function during acute exacerbation of COPD, by US.

Methods: This single-center, prospective study included patients with acute exacerbation of COPD symptoms. US measurements were performed within $72 \mathrm{hrs}$ after exacerbation and after improvement of symptoms. Diaphragmatic excursion and its thickening fraction (TF) were measured as markers of diaphragmatic function. TF was calculated as (thickness at end inspiration - thickness at end expiration)/thickness at end expiration.

Results: Ten patients were enrolled. All patients were male, and the mean age was 79.8 years. The TF of the right diaphragm showed a significant increase from the initial to the follow-up values $(80.1 \pm 104.9 \mathrm{~mm}$ vs. $159.5 \pm 224.6 \mathrm{~mm}, \mathrm{p}=0.011)$; however, the diaphragmatic excursion did not vary significantly between the initial and follow-up values (22 $\pm 6 \mathrm{~mm}$ vs $23 \pm 12 \mathrm{~mm}$ ). The change in excursion between the stable and exacerbation periods was positively correlated with time to the next exacerbation and negatively correlated with the time taken to recover from the exacerbation.

Conclusion: These data support the possibility that a defect in diaphragm thickening is related to acute exacerbation of COPD.

Keywords: ultrasonography, diaphragmatic excursion, diaphragmatic thickening, exacerbation, chronic obstructive pulmonary disease

\section{Introduction}

Chronic obstructive pulmonary disease (COPD) is characterized by persistent respiratory symptoms and chronic airflow limitation due to small airway disease and parenchymal destruction. Patients with COPD often experience sudden aggravation of respiratory symptoms, known as acute exacerbation of COPD. As COPD exacerbation leads to deterioration in quality of life and subsequent morbidity and mortality, the recently revised Global Initiative for Chronic Obstructive Lung Disease (GOLD) guidelines include history of acute exacerbation as a parameter for COPD assessment. ${ }^{1}$

The diaphragm is the main muscle for inspiration, and its importance in COPD development has long been suggested. ${ }^{2,3}$ Impairment of diaphragmatic function may be one of the main pathophysiological mechanisms in COPD and appears to be related to acute exacerbation. During acute exacerbation, biologic factors related to systemic
Correspondence: Choon-Taek Lee Division of Pulmonology and Critical Care Medicine, Department of Internal Medicine, Seoul National University College of Medicine, 82, Gumi-ro I73beon-gil, Bundang-gu, Seongnam-si, Gyeonggi-do, Seongnam, Seoul, South Korea

Tel +82 3I 7877002

Fax +82317874052

Email ctlee@snubh.org
International Journal of Chronic Obstructive Pulmonary Disease 2019:14 2479-2484

2479

DovePress $f y$ in $\boldsymbol{v}$ 
inflammation, prolonged use of steroids, and hyperinflation of the lungs may act as the main mechanisms leading to diaphragmatic dysfunction. ${ }^{4}$

The recent introduction of ultrasonography (US) allows non-invasive, bedside investigation of diaphragmatic function. US has been used to assess diaphragmatic function in patients with stable COPD, and a negative correlation has been found between diaphragmatic motion and dyspnea. ${ }^{5}$ However, the role of the diaphragm during exacerbation is not well investigated. In this pilot study, we aimed to investigate diaphragmatic function measured by chest US in patients with COPD, during acute exacerbation and post recovery.

\section{Methods}

\section{Study Design And Population}

This prospective study was conducted at a single tertiary hospital in Korea over a 15-month period (from January 2015 to March 2016). Patients aged $\geq 18$ years who were hospitalized through the emergency department or outpatient clinic for acute exacerbation of COPD were eligible for inclusion. The diagnosis of COPD was based on the GOLD guidelines. Acute exacerbation of COPD was defined as "an acute event characterized by a worsening of the patient's respiratory symptoms that is beyond normal day-to-day variation and leads to a change in medication". 6 Treatment of the exacerbation was at the discretion of the attending physician and followed current practice guidelines. All patients had undergone a pulmonary function test previously and had a ratio of forced expiratory volume in one second (FEV1) to forced vital capacity (FVC) of less than 0.7. All patients were $>40$ years old. Exclusion criteria were any of the following: presence of pulmonary diseases besides COPD, such as pleural effusion, pneumothorax, phrenic nerve palsy, and interstitial lung disease; medical history of chemical pleurodesis, neuromuscular disease, or chest wall deformities; known pregnancy; and/or severe exacerbation of COPD requiring immediate endotracheal intubation. This study was approved by the Institutional Review Board (IRB) of Ethics Committee of Seoul National University Bundang Hospital (IRB No.: B-1406/ 255-005). All participants provided written informed consent. The study was conducted in accordance with the applicable version of the Declaration of Helsink ${ }^{7}$.

\section{US Measurement}

Both diaphragmatic excursion and change in muscle thickening were measured using US to assess diaphragmatic function. US measurement was performed within $72 \mathrm{hrs}$ after exacerbation and 2 weeks after discharge by the same experienced respiratory physician who has performed more than 30 lung US evaluations. The diaphragm was examined using a B-mode US device (Logiq ${ }^{\circledR}$ e machine, GE Healthcare, Wauwatosa, WI, USA) connected to a 4$10-\mathrm{MHz}$ linear transducer. Patients were examined in the supine position, with an average inclination of 45 degrees. In the M-mode, diaphragmatic excursion (cm) was measured with the probe placed over one of the lower intercostal spaces in the anterior axillary lines and the liver serving as an acoustic window (Figure 1). Excursion amplitude was measured on the vertical axis tracing from the baseline to the point of maximum height of inspiration on the graph, as previously described. ${ }^{8,9}$ Diaphragm thickness (DT) was imaged at the zone of apposition of the diaphragm located between the midaxillary and posterior axillary lines and measured at end inspiration and end expiration. The percent change in DT between end expiration and end inspiration (DT fraction, DTF) was calculated as follows (DT at end inspiration - DT at end expiration)/ DT at end expiration $\times 100$. US examinations were performed thrice on both sides of the diaphragm in each patient. The value best representative of the diaphragmatic function was then recorded for analysis.

\section{Outcome Measurements}

Primary outcome was the change in diaphragmatic function as measured by US during acute exacerbations in COPD patients. Time until subsequent exacerbation and

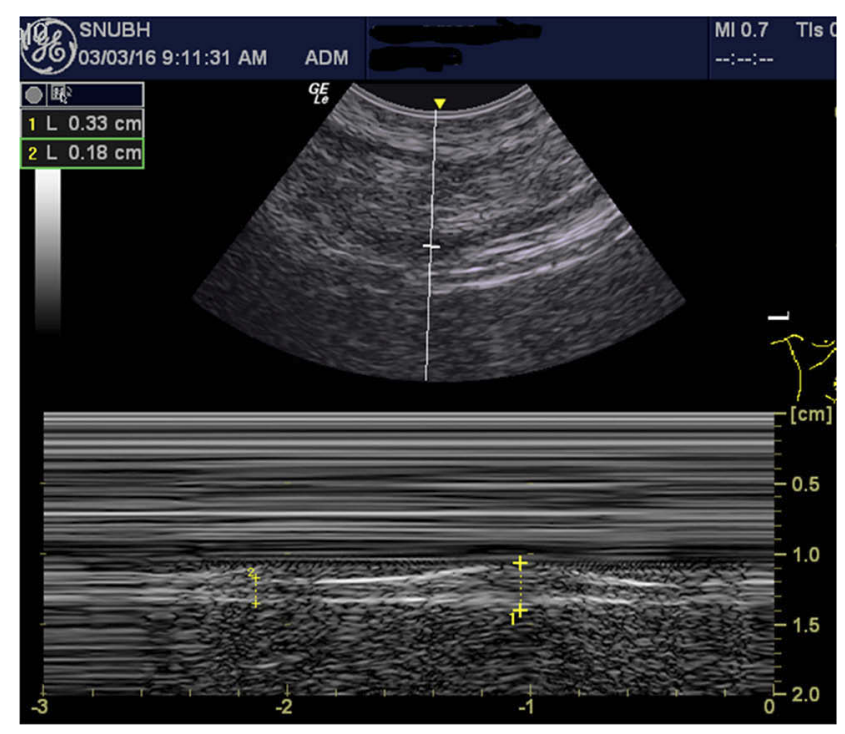

Figure I Right diaphragm thickness on B-mode ultrasonography. 
exacerbation duration were recorded as additional outcomes. Time to the onset of the next exacerbation was defined as the number of days from the onset of one exacerbation to the onset of the next exacerbation. Patients were asked at all visits if they had experienced recent exacerbations, hospitalization, or health care use. Exacerbation duration (time taken to recover from the exacerbation) was defined as the number of days for which the increased respiratory symptoms remained after exacerbation onset. Recovery from exacerbation was regarded as the first three or more consecutive symptomfree days.

\section{Statistical Analysis}

Continuous variables were expressed as medians and interquartile range (IQR 25-75\%), and categorical variables were expressed as frequencies and percentages. Chisquared test, Fisher's test, and Mann-Whitney $U$-test were used for analyses. Comparisons between paired groups were performed using Wilcoxon signed rank test. Correlations were assessed using Spearman correlation test. A p value $(\mathrm{p})<0.05$ was considered statistically significant. All analyses were performed using R software (version 3.3.2; http://www.R-project.org).

\section{Results}

In total, 14 patients with acute exacerbation of COPD were enrolled during the study period. Of these, 10 patients were included in this analysis; one patient was excluded because of the need for invasive mechanical ventilation after enrollment and three patients were excluded because of withdrawal of consent. All patients were male, and the mean age was 79.8 years. The average post-bronchodilator FEV1\% predicted value was $55.8 \%$, and the mean St George's Respiratory Questionnaire (SGRQ) score at screening was 44.7. Baseline characteristics of the study participants are presented in Table 1.

The right DTF was significantly decreased during COPD exacerbation as compared to that in the stable phase. The left DTF was also decreased during exacerbation; however, the difference was not statistically significant (Table 2). In contrast, diaphragmatic excursion on both the left and right sides did not show significant difference between the exacerbation and stable phases.

There was a strong correlation between DTF in the stable phase and baseline FEV1\% predicted value $(\mathrm{r}=0.89, \mathrm{p}=0.017$; Table 3$)$. No correlation was observed between diaphragmatic excursion, either during or after
Table I Characteristics Of The Study Population

\begin{tabular}{|l|l|}
\hline Characteristics & $\mathbf{N}=10$ \\
\hline Age, years & $79.8 \pm 8.1$ \\
Sex (male) & $10 / 10(100 \%)$ \\
Length of hospital stay, days & $7.6 \pm 3.3$ \\
Height & $1.66 \pm 0.04$ \\
Weight & $60.9 \pm 10.4$ \\
BMI & $22.3 \pm 3.8$ \\
FEVI, liters (predicted \%) & $55.8 \pm 25.5(55.8 \%)$ \\
FVC, liters (predicted \%) & $79.1 \pm 22.1(79.1 \%)$ \\
FEVI/FVC, \% & $44.8 \pm 12.6$ \\
RV, liters & $2.3 \pm 0.2$ \\
TLC, liters & $5.2 \pm 0.6$ \\
SGRQ, symptom & $43.0 \pm 21.9$ \\
SGRQ, activity & $65.5 \pm 21.1$ \\
SGRQ, impact & $32.7 \pm 15.9$ \\
SGRQ, total & $44.7 \pm 14.9$ \\
\hline
\end{tabular}

Abbreviations: BMI, body mass index; FEVI, forced expiratory volume in I s; FVC, forced vital capacity; RV, residual volume; TLC, total lung capacity; SGRQ, St. George's Respiratory Questionnaire.

Table 2 Ultrasound Parameters For Diaphragmatic Function

\begin{tabular}{|l|l|l|l|}
\hline & Initial & Follow-Up & p-value \\
\hline TF, right & $80.1 \pm 104.9$ & $159.5 \pm 224.6$ & 0.011 \\
TF, left & $81.9 \pm 53.2$ & $126.8 \pm 125.8$ & 0.173 \\
Excursion, right $(\mathrm{cm})$ & $2.2 \pm 0.6$ & $2.3 \pm 1.2$ & 0.753 \\
Excursion, left $(\mathrm{cm})$ & $1.4 \pm 0.6$ & $1.5 \pm 0.6$ & 0.345 \\
\hline
\end{tabular}

Abbreviation: TF, thickening fraction.

exacerbation, and FEV1\% predicted value at baseline. Additionally, there was no significant correlation between DTF and time to the next exacerbation. However, the degree of change in the diaphragmatic excursion between the exacerbation and stable phases was positively correlated with time until the next exacerbation (Figure 2).

\section{Discussion}

In this prospective study, we demonstrated that DTF measurements based on diaphragmatic US assessment were significantly reduced in patients with COPD during acute exacerbations compared to that post recovery after exacerbation. However, diaphragmatic excursion as assessed by M-mode US did not show significant difference between the exacerbation and stable phases.

Measurement of diaphragmatic function with US has become widely used in patients with neuromuscular disorder or acute respiratory failure because of its easy applicability and noninvasiveness. Although, the gold standard method for quantifying diaphragmatic function 
Table 3 Pulmonary Function Test Variables In Correlation With Diaphragmatic Function

\begin{tabular}{|l|l|l|l|l|}
\hline & FEVI, Liters & FEVI, Predicted \% & FVC, Liters & FVC, Predicted \% \\
\hline Initial (during exacerbation phase) & & & & \\
$\quad$ TF, right & $0.289(0.450)$ & $0.438(0.238)$ & $0.086(0.827)$ & $0.166(0.669)$ \\
TF, left & $0.362(0.338)$ & $0.309(0.419)$ & $0.082(0.834)$ & $0.112(0.774)$ \\
Excursion, right (cm) & $0.430(0.395)$ & $0.010(0.985)$ & $0.586(0.222)$ & $0.199(0.706)$ \\
Excursion, left (cm) & $0.438(0.385)$ & $0.357(0.488)$ & $0.905(0.013)^{\mathrm{a}}$ & $0.814(0.049)^{\mathrm{a}}$ \\
\hline Follow-up (during stable phase) & & & & \\
TF, right & $0.512(0.159)$ & $0.892(0.017)^{\mathrm{a}}$ & $0.394(0.294)$ & $0.495(0.175)$ \\
TF, left & $0.687(0.04 \mathrm{I})^{\mathrm{a}}$ & $0.744(0.022)^{\mathrm{a}}$ & $0.441(0.234)$ & $0.388(0.303)$ \\
Excursion, right (cm) & $0.206(0.696)$ & $0.293(0.573)$ & $0.584(0.224)$ & $0.100(0.850)$ \\
Excursion, left (cm) & $0.564(0.244)$ & $0.308(0.552)$ & $0.856(0.030)^{\mathrm{a}}$ & $0.615(0.194)$ \\
\hline
\end{tabular}

Notes: Values are expressed as correlation coefficient, $r$ value ( $p$-value); ${ }^{a} \mathrm{P}<0.05$.

Abbreviations: FEVI, forced expiratory volume in I s; FVC, forced vital capacity; TF, thickening fraction.

is the measurement of the negative pressure generated by diaphragm contraction in response to phrenic nerve stimulation, ${ }^{10}$ US measurement of DT, excursion, and DTF has been proposed for estimating diaphragmatic function. Recently, a systematic review was presented by Zambon et al on the role of US in assessing diaphragmatic
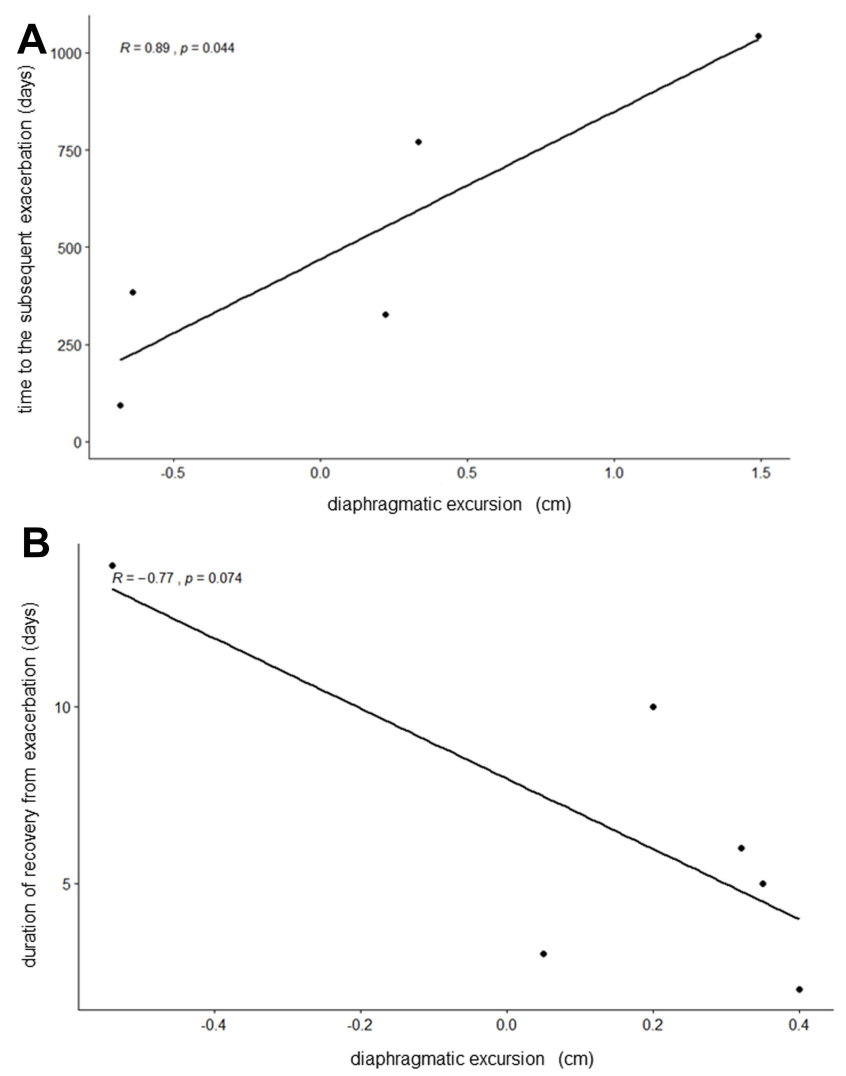

Figure $\mathbf{2}$ Linear correlation between the change in diaphragmatic excursion on the right and (A) time to subsequent exacerbation and (B) time taken to recover from the exacerbation. function in critically ill patients. ${ }^{11}$ The authors concluded that US could be a useful tool to assess diaphragmatic dysfunction in critically ill patients.

Additionally, diaphragmatic function has also been examined using US in patients with COPD, and these patients present with a higher rate of diaphragmatic dysfunction in contrast to age- and sex-matched healthy control individuals. ${ }^{12}$ Davachi et al compared 25 participants with COPD and 25 healthy controls, and detected statistically significant differences in terms of diaphragmatic motility, as measured by US, between the two groups. ${ }^{13}$ Moreover, Elsawy reported that DTF was significantly decreased in the COPD group and decreased with the increasing severity of COPD. ${ }^{14}$

To date, diaphragmatic US has been examined mainly in stable COPD patients and not in patients with acute exacerbation of COPD. We found that diaphragm contractility, as measured by DTF, was lower during the exacerbation period than during the stable period. In a recently published pilot study, Antenora et al performed diaphragmatic US in patients with acute exacerbation of severe COPD before starting noninvasive ventilation. Severe diaphragmatic dysfunction was seen in almost one-quarter of the patients; ${ }^{15}$ our results are consistent with these findings. The pathological mechanism underlying diaphragmatic dysfunction during acute exacerbation is multifactorial. The synergistic effect of mechanical disadvantage, functional exhaustion, systemic inflammation, and pharmacological damage (steroid-induced) might cause diaphragmatic dysfunction during acute exacerbation. ${ }^{12}$

We examined both DTF and diaphragmatic excursion; however, diaphragmatic excursion did not show significant differences between the stable and exacerbation phases. As 
movement of the diaphragm is largely determined by spontaneous breathing in patients, various factors, including high respiratory rate and dynamic obstruction, could have influenced diaphragmatic excursion. Previous studies have also demonstrated the usefulness of DT, and not excursion, in assessing diaphragmatic function in patients with acute respiratory failure. ${ }^{12,16,17,18}$

Previously, we demonstrated that both the right and left DTF were decreased during exacerbation; however, the change in the left DTF did not reach statistical significance. Unlike the thickening fraction of the right hemidiaphragm, using the liver as an ultrasound window, interference of gastric air with the ultrasound might result in inaccurate measurement of the thickening fraction of the left hemidiaphragm. Similar to these findings, Boussuges et al successfully measured maximal diaphragmatic excursion on the right hemidiaphragm in all patients in their study; however, they were able to perform this assessment on the left hemidiaphragm in only 45 out of 210 patients $(23 \%){ }^{9}$ Another prospective study showed that lung function parameters were highly correlated with diaphragmatic dysfunction as measured by US on the right hemidiaphragm. ${ }^{19}$

Another important result of our study is the strong positive correlation between the change in excursion and time to the next exacerbation. We also found that the change in excursion was negatively correlated with the time taken to recover from the exacerbation. Patients who showed more improvement in diaphragmatic excursion after exacerbation needed shorter time to recover from the exacerbation and would have a longer stable period until the next exacerbation. Thus, failure in improvement of diaphragmatic excursion during recovery could be a prognostic marker for frequent exacerbations.

Our study has a few limitations. First, the small sample size can raise some concerns. Our results, however, are significant despite the small sample analyzed, and should be considered when designing future studies with more patients. Second, this study was conducted at a single center; hence, the data should be confirmed in larger samples and multiple centers. A third limitation is the absence of transdiaphragmatic pressure measurements via esophageal or gastric transducer after phrenic nerve stimulation, which are accepted as the ideal standards for assessment of diaphragmatic function. However, US measurement of diaphragmatic function has previously been validated in patients with neuromuscular disorders, ${ }^{20}$ and it has been widely studied in patients with COPD or interstitial lung disease, particularly intubated patients being prepared for weaning and extubation. ${ }^{8,11}$ Finally, in our study, measurements were performed during spontaneous breathing in an exacerbation setting without any assessment of lung volumes.

In conclusion, our pilot study shows that diaphragmatic dysfunction occurs during acute exacerbation of COPD, and patients showing greater improvement of function during recovery will likely have delayed appearance of future exacerbations. US, as a tool for non-invasive assessment of diaphragmatic function, could play a practical role in the management of patients with acute exacerbation of COPD.

\section{Acknowledgment}

This research was supported by a grant from the Seoul National University Bundang Hospital, Republic of Korea (grant no. 02-2012-043).

\section{Author Contributions}

All authors made substantial contributions to conception and design, acquisition of data, or analysis and interpretation of data; took part in drafting the article or revising it critically for important intellectual content; gave final approval of the version to be published; and agree to be accountable for all aspects of the work.

\section{Disclosure}

The authors report no conflicts of interest in this work.

\section{References}

1. Vogelmeier CF, Criner GJ, Martinez FJ, et al. Global strategy for the diagnosis, management and prevention of chronic obstructive lung disease 2017 report: GOLD executive summary. Respirology. 2017;22(3):575-601.

2. Ottenheijm CA, Heunks LM, Dekhuijzen PN. Diaphragm muscle fiber dysfunction in chronic obstructive pulmonary disease: toward a pathophysiological concept. Am J Respir Crit Care Med. 2007;175 (12):1233-1240. doi:10.1164/rccm.200701-020PP

3. Ottenheijm CA, Heunks LM, Dekhuijzen RP. Diaphragm adaptations in patients with COPD. Respir Res. 2008;9:12. doi:10.1186/14659921-9-12

4. Gayan-Ramirez G, Decramer M. Mechanisms of striated muscle dysfunction during acute exacerbations of COPD. J Appl Physiol (1985). 2013;114(9):1291-1299. doi:10.1152/japplphysiol.00847.2012

5. Paulin E, Yamaguti WP, Chammas MC, et al. Influence of diaphragmatic mobility on exercise tolerance and dyspnea in patients with COPD. Respir Med. 2007;101(10):2113-2118. doi:10.1016/j.rmed.2007.05.024

6. Celli BR, MacNee W. Standards for the diagnosis and treatment of patients with COPD: a summary of the ATS/ERS position paper. Eur Respir J. 2004;23(6):932-946. doi:10.1515/9783110208856.233

7. World Medical Association. Declaration of Helsinki: ethical principles for medical research involving human subjects. Available from: https:// www.wma.net/policiespost/wma-declaration-of-helsinki-ethical-princi ples-for-medical-research-involving-human-subjects/. 
8. Matamis D, Soilemezi E, Tsagourias M, et al. Sonographic evaluation of the diaphragm in critically ill patients. Technique and clinical applications. Intensive Care Med. 2013;39(5):801-810. doi:10.1007/ s00134-013-2823-1

9. Boussuges A, Gole Y, Blanc P. Diaphragmatic motion studied by m-mode ultrasonography: methods, reproducibility, and normal values. Chest. 2009;135(2):391-400. doi:10.1378/chest.08-1541

10. American Thoracic Society/European Respiratory Society. ATS/ERS Statement on respiratory muscle testing. Am J Respir Crit Care Med. 2002;166(4):518-624.

11. Zambon M, Greco M, Bocchino S, Cabrini L, Beccaria PF, Zangrillo A. Assessment of diaphragmatic dysfunction in the critically ill patient with ultrasound: a systematic review. Intensive Care Med. 2017;43(1):29-38. doi:10.1007/s00134-016-4524-z

12. Marchioni A, Castaniere I, Tonelli R, et al. Ultrasound-assessed diaphragmatic impairment is a predictor of outcomes in patients with acute exacerbation of chronic obstructive pulmonary disease undergoing noninvasive ventilation. Crit Care. 2018;22(1):109. doi:10.1186/s13054-018-2033-X

13. Davachi B, Lari SM, Attaran D, et al. The relationship between diaphragmatic movements in sonographic assessment and disease severity in patients with stable Chronic Obstructive Pulmonary Disease (COPD). J Thorac. 2014;2(3):187-192.

14. Elsawy SB. Impact of chronic obstructive pulmonary disease severity on diaphragm muscle thickness. Egypt J Chest Dis Tuberc. 2017;66 (4):587-592. doi:10.1016/j.ejcdt.2017.08.002
15. Antenora F, Fantini R, Iattoni A, et al. Prevalence and outcomes of diaphragmatic dysfunction assessed by ultrasound technology during acute exacerbation of COPD: A pilot study. Respirology. 2017;22 (2):338-344. doi:10.1111/resp.12916

16. Umbrello M, Formenti P, Longhi D, et al. Diaphragm ultrasound as indicator of respiratory effort in critically ill patients undergoing assisted mechanical ventilation: a pilot clinical study. Crit Care. 2015;19:161. doi:10.1186/s13054-015-0894-9

17. Goligher EC, Fan E, Herridge MS, et al. Evolution of diaphragm thickness during mechanical ventilation. Impact of Inspiratory Effort. Am J Respir Crit Care Med. 2015;192(9):1080-1088. doi:10.1164/ rccm.201503-06200C

18. Vivier E, Mekontso Dessap A, Dimassi S, et al. Diaphragm ultrasonography to estimate the work of breathing during non-invasive ventilation. Intensive Care Med. 2012;38(5):796-803. doi:10.1007/ s00134-012-2547-7

19. Scheibe N, Sosnowski N, Pinkhasik A, Vonderbank S, Bastian A. Sonographic evaluation of diaphragmatic dysfunction in COPD patients. Int J Chron Obstruct Pulmon Dis. 2015;10:1925-1930. doi: $10.2147 /$ COPD.S85659

20. Whitehead NP, Bible KL, Kim MJ, Odom GL, Adams ME, Froehner SC. Validation of ultrasonography for non-invasive assessment of diaphragm function in muscular dystrophy. $J$ Physiol. 2016;594 (24):7215-7227. doi:10.1113/JP272707

\section{Publish your work in this journal}

The International Journal of COPD is an international, peer-reviewed journal of therapeutics and pharmacology focusing on concise rapid reporting of clinical studies and reviews in COPD. Special focus is given to the pathophysiological processes underlying the disease, intervention programs, patient focused education, and self management protocols. This journal is indexed on PubMed Central, MedLine and CAS. The manuscript management system is completely online and includes a very quick and fair peer-review system, which is all easy to use. Visit http://www.dovepress.com/testimonials.php to read real quotes from published authors. 